\section{Should Pregnant Women Care for AJDS Patients?}

To the Editor:

The article on "Caring for the Infectious Patient: Risk Factors During Pregnancy" by Gurevich and Tafurol was generally a good review.

It should be noted that patients with rubella are on contact precautions for those using categories, and urine, respiratory secretion precautions for disease-specific. The authors in one paragraph call for Enteric Precautions and shortly thereafter recommend Respiratory. Neither is correct.

I believe it is excessive to exclude all pregnant women from caring for patients with AIDS. Firstly, it has not been demonstrated that nurses are at higher risk of CMV infection and secondly patients with AIDS can be screened for CMV so that at least a decision can be made considering CMV rather than AIDS.

\section{REFERENCES}

1. Gurevich I. Tafuro P: Caring for the infectious patient: Risk factors during pregnancy. Infect Control 5:482-488

Patricia Solenberger, RN, MA Infection Control Practitioner Crouse Irving Memorial Hospital Syracuse, New York

The author of the article in question responds to Ms. Solenberger's comments.

Some of Ms. Solenberger's points are well-taken, others require some discussion.

There are three issues here. The first concerns the care of patients with postnatal rubella. Under the new guideline developed by the CDC, patients with rubella should be on Contact "Isolation" (not Ms. Solenberger's Contact "Precautions"), a term which we find confusing and which motivated us to use the old Respiratory Isolation for which a mask is required to enter the room. The new isolation requires a mask only if coming as close as 3 feet to the patient. Even if one does not initially intend to come that close, one can not always foresee what may actually occur in a room that may necessitate closer contact (or the patient may sneeze and thus droplets can reach 5 feet away). Therefore, a mask is not an unreasonable measure on entering the room. Since handwashing should usually occur after handling urine, or leaving the room of a patient on Respiratory Isolation, we do not find the use of gloves indicated.

Issue two, not discussed at all in our article, is handling of a newborn with congenital rubella. Such infants should be cared for under the old Strict Isolation or the new Contact Isolation, because of the concern with shedding virus from secretions and excretions.

Third is our concern about such a child if admitted to a hospital at a later time (up to 20 months), who may still be asymptomatically shedding virus from the pharynx and the urine, unless cultures at 3 months were already negative. Here, the precautionary measures require only gloves and handwashing, covered by the old Secretion Precautions (Enteric Precautions was used in error).

We do not care to use the new Contact Isolation, since we use private rooms only for patients with diseases where airborne spread is anticipated. However, each institution must make its own determination, as is also true for our recommendation regarding CMV.

We agree that nurses, in general, are at no greater risk of CMV acquisition than women in general. But the risk to the fetus, however small, carries such grave consequences that we do not believe many people would insist that pregnant nurses care for CMV shedders. Since AIDS and CMV are so closely linked, and even those patients who are not presently shedding may reactivate their $C M V$ infection, we stand by our recommendation. Also, pregnant employees are not automatically excluded from contact with AIDS patients. They are only given the opportunity to decline this patient assignment, which is not the case for most other patients with communicable diseases.

Inge Gurevich, RN, MA Infection Control Practitioner Nassau Hospital Mineola, New York

\section{Filter Uso- What It Can and Cannot Prevent}

\section{To the Editor:}

In answer to Ms. Tanking's query in Infection Control November 1984, regarding IV filter use, the answers given by Ms. Crow are essentially correct. ' Further information is available in an article written for the National Intravenous Therapy Association called "IV Filters: A Standard of Care." 2

In it, I explain the disadvantages and danger of IV filters as well as what they can and cannot prevent. The projected cost of their use is in the millions of dollars, which can be used in more productive ways-for example, by hiring more IV therapists to render better IV care. Copies are available from the author.

\section{REFERENCES}

1. Crow S: IV Therapy options, letter to the editor response. Infect Control 5:509.

2. Gurevich I: IV filters: A standard of care. National Intravenous Therapy Journal 7:399.

Inge Gurevich, RN, MA Infection Control Practitioner Nassau Hospital Mineola, New York 